This item was submitted to Loughborough's Research Repository by the author.

Items in Figshare are protected by copyright, with all rights reserved, unless otherwise indicated.

\title{
Enabling urban-scale energy modelling: a new spatial approach
}

PLEASE CITE THE PUBLISHED VERSION

http://dx.doi.org/10.1080/09613218.2013.813169

PUBLISHER

(c) Taylor and Francis

VERSION

AM (Accepted Manuscript)

LICENCE

CC BY-NC-ND 4.0

REPOSITORY RECORD

Taylor, Simon, Denis Fan, and Mark Rylatt. 2019. "Enabling Urban-scale Energy Modelling: A New Spatial Approach". figshare. https://hdl.handle.net/2134/13075. 
This item was submitted to Loughborough's Institutional Repository (https://dspace.lboro.ac.uk/) by the author and is made available under the following Creative Commons Licence conditions.

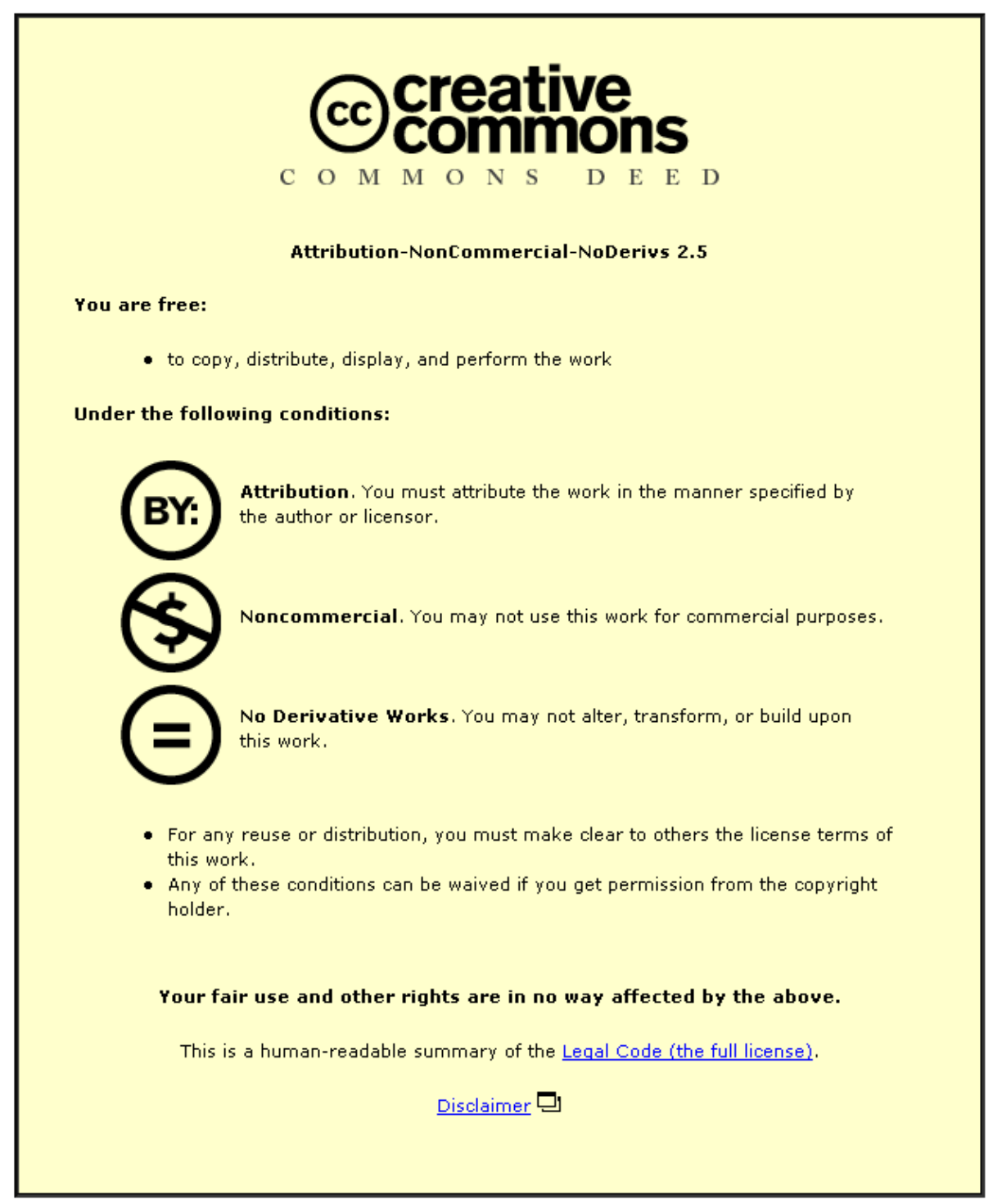

For the full text of this licence, please go to: http://creativecommons.org/licenses/by-nc-nd/2.5/ 
ENABLING URBAN SCALE ENERGY MODELLING:

A NEW SPATIAL APPROACH

Simon Taylor ${ }^{1,}$, Denis Fan ${ }^{2}$ and Mark Rylatt ${ }^{3}$

1: School of Civil and Building Engineering, Loughborough University, LE1 3TU, UK, s.c.taylor@lboro.ac.uk

2: Department of Energy and Climate Change, 3 Whitehall Place, London SW1A 2AW, UK, denis.fan@decc.gsi.gov.uk

3: Institute of Energy and Sustainable Development, De Montfort

University, Leicester, LE1 9BH, UK, rylatt@dmu.ac.uk

* Corresponding author 


\section{ENABLING URBAN SCALE ENERGY MODELLING:}

A NEW SPATIAL APPROACH

\section{Abstract}

Urban-scale energy modelling provides an ideal tool for studying nondomestic energy consumption and emissions reduction at the community level. In principle, an approach based on the characteristics of individual commercial premises and buildings is attractive but poses a number of challenges, the most immediate of which is deciding precisely what to model. For a range of reasons connected with their self-contained nature, individual non-domestic buildings would ideally be selected. However, the main information sources available, digital mapping and business taxation data, are not based on "buildings" and do not use the concept, thus making an automatic approach problematic. At the same time, manual identification of the distinct buildings in a city is not a practical proposition because of the numbers involved.

The digital mapping and business taxation data are brought together in the local land and property gazetteer (LLPG). An analysis of the relationships between the relevant elements, namely building polygons and premises attracting business taxation, allowed a unit to be defined that matches the definition of a "building" in most circumstances and can be applied without the need for human intervention. This novel approach provides a firmer basis for modelling non-domestic building energy at the urban scale. (198 words) 


\section{Keywords}

Urban, non-domestic, energy, spatial, modelling, city-scale. 


\section{Introduction}

Urban scale energy models based on the characteristics of commercial premises and buildings are becoming more attractive to policymakers in view of the great potential for energy and emissions reductions in this sector, which would make a significant contribution to the achievement of 2050 goals in the UK (HM Government, 2011). Top-down methods are considered inappropriate at this scale, because the attribution of aggregate data on which they depend does not support meaningful comparisons and they generally do not capture relevant technological and behavioural change. For the domestic stock, so-called bottom-up methods can be scaled up, e.g. Min et al (2010). Such an approach is based on the adaptation of relatively simple steady state models used for single dwellings, although dataset reduction is still potentially problematic. In the case of the non-domestic stock, however, the methods used for single buildings are far less readily adaptable. Even accepting that a fully dynamic modelling approach is currently unrealistic at this scale, simpler models (e.g. SBEM) still have massive data requirements.

An additional problem relates to the nature of the available data, described more fully in Section 3. A key determinant of non-domestic energy consumption is the activities carried out by the occupants (Mortimer et al, 2000a). The only comprehensive source of such information in the UK is business taxation data. This relates to premises, which have a complex 
relationship with buildings, making the integration of activity and physical building data highly problematical.

This paper deals with a fundamental aspect of the problem and is based on work on the 4M project (Lomas et al 2010, 4M 2013), which used the city of Leicester, UK as a case study. For this project a model has been developed, based closely on ISO 13790, which determines energy consumption primarily as a result of the balance between heat gains from occupants, electricity use and the heating system, and heat losses through the walls. The question addressed here is the scale at which the modelling should be carried out. In other words, a decision is needed on the element of the built environment upon which the calculations should be performed: business premises, individual buildings (if they can be defined clearly enough), blocks of adjoining buildings, or some other unit.

\section{Previous work}

There is little prior work specifically on the city-scale building energy modelling that is the subject of the present work, but it is instructive to consider briefly the approaches used from the national scale down so that some general principles and constraints can be better appreciated.

Pout (2000) used the Non-Domestic Energy and Emissions Model (NDEEM) to estimate the current end-use energy consumption of premises in the UK non-domestic stock. As in the present work, but at the national 
scale, N-DEEM also has the aim of predicting the cost-effectiveness of energy efficiency interventions and the potential for carbon savings. Floor areas, classified mainly by the activity types of building occupiers, are based on Bruhns et al (2000) grossed up to cover the whole of the UK. Energy end-uses are estimated for each of these classes and broken down by fuel type. As in the present approach energy consumption inputs for each activity are sourced mainly from the Sheffield Hallam University (SHU) energy surveys (Mortimer et al, 2000b). This survey was the largest of its kind ever undertaken in the UK but nevertheless did not provide sufficient data for all activity types. The problem of insufficient data plagues all attempts to model energy consumption on a large scale and $\mathrm{N}$ DEEM resorts to reasoning from similar activity types to fill such gaps. At this scale understandably no attempt is made to model individual buildings and the estimates of annual consumption relate entirely to premises.

The Carbon Reduction in Buildings (CaRB) project developed a nationalscale model (Bruhns et al, 2006) to estimate the end-use energy consumption of the non-domestic building stock. Despite its low resolution it is a "bottom-up" model (in building energy rather than general energy modelling terms), using data on individual premises, and is also related to the approach described in this paper through its reliance on UK Valuation Office Agency data and its use of floor areas - for individual premises rather than buildings - derived from this source. 
The Non-Domestic Carbon Scenario Model (NDCSM) of Hinnells et al (2008) estimates the energy consumption and carbon emissions for the non-domestic stock in relation to climate change intervention scenarios. Energy data is again sourced from the SHU surveys, despite some caveats from the authors that it is not fully representative of the building stock and not sufficiently detailed in relation to energy end-uses. To some extent this model relies on the CaRB-based model for gross floor area and uses activity and floor area data inputs from the VOA - via the CaRB stock model - and is evidently applicable only at the national scale.

Although not confined to non-domestic buildings and premises, the Energy and Environmental Prediction (EEP) model (Jones et al, 2000) can be used to predict the energy consumption and carbon emissions related to this sector at a scale more comparable to the approach described in this paper. It also shares the basic technique of deriving floor areas from a GIS but requires external surveys of buildings to acquire data such as number of storeys. Again the approach is activity-based and in view of the claimed potential for modelling down to individual postcode level would appear to require a reliable methodology to address the problem of integrating spatial and non-spatial data sources data sources as now proposed, but this is not elucidated. References to a statistical clustering technique suggest in fact that the minimum satisfactory resolution must be much coarser, but reliance on external surveys presumably rules out modelling at the city scale. 
Outside the UK there are more examples of approaches that can be used at or below the city scale. Such models tend to be shaped very much by data availability and type, and local building characteristics, but do allow some additional perspective on the field in the UK. In the USA, Huang et al (1991) developed a model based on a system of non-domestic building archetypes, each assigned averaged characteristics derived from a number of data sources grossed up to estimate the energy demand of metropolitan districts across the USA, with the specific purpose of assessing suitability for cogeneration schemes. A major data source was the Non-residential Building Energy Consumption Survey (NBECS), which preceded the Commercial Buildings Energy Consumption Survey (CBECS), but inadequate and unrepresentative data was clearly a problem and expert "guesstimates" were resorted to where necessary. Huang and Brodrick (2000) describe an application of the same model to predict the effects on energy consumption of technology interventions across the entire US building stock. Rather broad building age categories - just two - were used, which would not reflect the relatively frequent changes in building regulations in the UK for example. However this body of work is interesting in its attempt to integrate data on built form, energy end-use and activity types.

Yamaguchi et al. (2007) describe a model using a method called "district clustering" designed to identify archetypal urban areas, which can be aggregated to represent a city. It relies on surveys of larger buildings to 
establish the archetypes but the rationale for selecting areas and building is not entirely clear and is unlikely to translate well internationally.

\section{Data Sources}

In the UK, two data sources are available that are potentially valuable as enablers of non-domestic building energy modelling at the city scale: the Ordnance Survey digital MasterMap data (OS 2013), which depict the outlines of all buildings and parts of buildings as polygons known as building TOIDs ("bTOIDs" hereafter); and the Valuation Office Agency (VOA) Rating List of premises (strictly, hereditaments) attracting business taxation ("premises"). Although there is a drive to link these two datasets at national level so that premises can be geo-referenced straightforwardly, at the time of writing this effort remains very partial. There may therefore be an initial challenge to relate the addresses of premises in the VOA dataset to map objects and this must be achieved before the datasets can be integrated to give useful information. In the case of Leicester - as will be described below - this process is already largely complete. Commercial and research software is available to automate the linkage of these datasets where necessary, although this approach is unlikely to deliver the accuracy or completeness of manual exercises, and they are not the focus of this paper. The main challenge is to establish the basic modelling unit.

If bTOIDs represented the building footprint in an invariant one-to-one relationship, they would provide the energy modeller with a straightforward 
approach. However, often buildings comprise several bTOIDs. This is at least partly due to the intention (OS 2001, p. 74ff) to represent different types of construction, although in some cases the division into separate bTOIDs appears arbitrary. One consequence is the significant excess of bTOIDs in the analysis described later.

The VOA Rating List includes the great majority of non-domestic premises, and most of them appear in the more detailed Summary Valuation (SMV) database which provides the taxable (roughly, occupied) area, overall function (e.g. office or shop) and for larger premises some breakdown by room or floor of the use of space. It seems unlikely that this "subpremises data" could be related reliably to buildings or parts of buildings, and so the unit of interest in this dataset is the premises, with the further detail available in the subpremises data enabling the determination of average premises properties.

In order for non-domestic energy consumption modelling to be carried out, the two main data sources, MasterMap and VOA, need to be brought together in such a way that they apply to the same space. Fortunately for the present work, much of this linking has already been carried out for Leicester as part of the creation by Leicester City Council of the Local Land and Property Gazetteer (LLPG). For the moment we have taken this linking as correct and have studied it in detail. The results shed light on the subject of the present paper, and so the analysis is presented separately in a later section. In summary, data on the following are available: 
1. All the built structures in the city, both domestic and non-domestic (from MasterMap)

2. The non-domestic premises (from the VOA)

3. The linkages between the two (from the LLPG).

Item 1 provides wall, roof and ground areas and orientation. A related dataset contains the heights of the structures associated with the bTOIDs expressed as a "2.5D model", obtained by combining the bTOID polygons with LIDAR data supplied by Infoterra, UK.

Item 2 provides the taxable area, the non-domestic function (shop, office, etc.) and, in most cases, some breakdown of space use. Analysis of the VOA data (Liddiard et al 2010, Liddiard 2012) has assigned some operational details, e.g. internal temperatures and electricity consumption, to the premises in item 2.

\section{The self-contained unit}

4.1 Requirements for the modelling unit

An important question to address is the scale at which the modelling should be carried out. Some of the requirements can be identified fairly straightforwardly. Whatever unit is chosen, it must be amenable to modelling. For the approach adopted, this means being bounded by a well-defined (though possibly not well understood) building envelope and having consistent properties so that the less well known properties (e.g. 
thermal capacity) can be deduced from others, such as age or construction method, and so that assumptions about building material and glazing in one wall are applicable to others.

Since the aim of the modelling is to understand energy consumption, the unit should have its own energy metering so that the characteristics of its energy use are correctly represented (Neffendorf et al 2009). These points correspond to a requirement for being self-contained and so are consistent with a further requirement that it be meaningful to consider the unit in isolation. The unit should be small enough to allow a reasonable degree of granularity, so that the results can be analysed and presented in different ways. There should ideally be no arbitrariness in the definition of the unit. Finally, the large quantity of data involved means that the unit should be straightforward to identify, work with and automate, with no requirement for human judgement.

So ideally the chosen unit, which will be referred to as the "self-contained unit" or SCU, should have the following properties:

1. Physically distinguishable or otherwise meaningful when considered in isolation

2. Appropriate for modelling:

2.1. Bounded by a single building envelope whose properties are known or can be deduced

2.2. Having consistent properties, probably due to having been built as a unit e.g. all walls having the same construction method and 
window type, thermal capacity consistent with date and type of construction, etc.

3. Self-contained (e.g. has its own energy metering)

4. Non-arbitrary

5. As small as possible consistent with the above, to allow a reasonable degree of granularity

6. Amenable to automation.

\subsection{Analysis}

Item 2.1 is probably the most important criterion. It means the SCU cannot be a premises, because a building envelope is not applicable. It also means that the SCU cannot be smaller than a bTOID or include parts of a bTOID because then there would be parts of the envelope for which there was no information. In some cases the SCU can be identified with a single bTOID, but not always, because some bTOIDs represent building subsections - parts of existing structures which are not self-contained and so do not satisfy item 3. If the SCU does consist of more than one bTOID, they must be in thermal contact in order for them to have a single building envelope. Item 2.1 also means that the unit should include all storeys in a multi-storey structure. To summarize this stage of the analysis, the SCU must consist of one or more bTOIDs in thermal contact.

The next stage is to decide which bTOIDs to include. One option would be to define the modelling unit as any set of contiguous bTOIDs. This has the advantage of clarity and simplicity. It provides a clear answer to the 
question about whether a terrace of five identical shops is five buildings or one - it is one. It takes care of bTOIDs representing building subsections automatically. It would also be consistent with the natural way to approach modelling a modern shopping centre: as a single building.

Where it does not work well is that in some cases (e.g. the row of shops referred to above) the unit will be large and will include many selfcontained structures, possibly of very different ages and therefore construction types and which almost certainly have their own heating systems. It would therefore not be consistent with requirements $2.2,3$ and 5. It would be preferable if the SCU corresponded to a building as normally understood, but this is problematic. There is no clear definition available (Neffendorf et al 2009) and in particular no obvious way of translating the available data (VOA and MasterMap) into units called "buildings". So as things stand it would be difficult to identify the bTOIDs comprising a building manually and impossible to do it automatically - and yet the large quantity of data in city-scale modelling (about 10,000 premises in Leicester) makes an automatic approach necessary.

A possible solution was developed by analysing the relationship between bTOIDs and premises, as captured in the LLPG. 


\section{Study of the local land and property gazetteer (LLPG)}

\subsection{Relationships between bTOIDs and premises}

The bTOID and premises data, from the OS MasterMap and VOA databases respectively, provide complementary information about spaces used for commercial and industrial purposes.

The bTOIDs represent buildings, or parts of them, which exist physically. For present purposes they have constant, uniform properties and are permanent. Premises, by contrast, are a construct used by government for taxation purposes. They exist only as entries in a database and are relatively variable and transient, strongly dependent on the economic climate. But they provide vital evidence of the way an organization uses space, and up to now this has been their primary role in research of this type, e.g. Steadman et al (2000).

With the introduction of the LLPG, the bTOIDs and premises relating to the same spaces have been linked, thus defining the bTOIDs within which an organization operates. This feature is now examined in more detail.

The previous section showed that the definition of an appropriate unit for modelling led to the requirement that it should be one or more bTOIDs in thermal contact. An additional requirement is now proposed: if two bTOIDs in thermal contact are associated with the same premises, both bTOIDs should form part of the same modelling unit. The logic is that attached bTOIDs that form part of the same premises are highly likely to be part of 
the same building as normally understood; and if that is not the case, there is still some commonality because the building is being operated by the same organization, lessening any errors due to the assumption.

This additional requirement provides a solution to the problem of how to deal with bTOIDs that represent building subsections. Provided they have been identified as being part of the same premises as the bTOID to which they are attached, the requirement will include them in the same modelling unit. The requirement also provides a natural distinction between two adjoining SCUs if they have no premises in common. The two requirements can now be combined to give a definition of the selfcontained unit applicable to the data in the LLPG. There are several possible equivalent statements of the definition. The one below is chosen because it is the most succinct and straightforward to understand, and at the same time focuses on the most important feature of the SCU: the boundary.

\section{An SCU is a set of contiguous bTOIDs with a continuous boundary defined by (a) external walls and (b) party walls between bTOIDs having no common premises.}

The application of the definition to some simple cases is illustrated schematically in Fig. 1. Each rectangular block represents a bTOID, and the curved loops in Fig. 1(a) represent the premises they contain. In the simplest case at the top left, a single bTOID contains a single premises. At 
the bottom left, a premises extends between two buildings, and at the bottom centre, two premises are contained in a single bTOID.

Figure 1 near here

Application of the SCU definition to Fig. 1(a) shows that the boundary of an SCU is defined by bTOID walls other than those party walls traversed by a loop. It follows that SCUs can be readily visualized by simply making such "common premises walls" less visually obvious than the others, as shown in Fig $1(b)$.

It turned out that application of the definition to real data was facilitated by some preliminary analysis of the database, which is described next.

\subsection{Analysis of the Leicester LLPG}

After removal of irrelevant records and minor corrections to a small number of entries, the LLPG could for the purposes of the present work be characterized as a two-column table. One column contains reference numbers to premises in the VOA; the other contains reference numbers to bTOIDs in MasterMap. A given row indicates a link between a premises and a bTOID, showing that at least some of that premises is contained within at least some of that bTOID.

Two features of the VOA Rating List meant that the LLPG needed to be modified for the planned task. First, as described later, preliminary work established that the SCU concept does not work well for shopping centres, 
so the related premises and bTOID records were removed. Second, places of religion (churches, mosques, etc.) are not included in the Rating List so LLPG records were created manually for such establishments. A further category of premises that the Rating List does not contain, Crown Estate properties, might in general need to be taken into account but no such properties exist in the city of Leicester (Crown Estate 2013).

After making the above changes there were 24,470 records in all, linking 10,862 premises to 16,358 bTOIDs. The large excess of bTOIDs over premises is a result of the finding referred to earlier, that structures that by any reasonable definition would be classed as buildings often consist of several bTOIDs, some of them very small (referred to as "building subsections" above). This alone shows that the bTOID is unsuitable as the unit of space for modelling: the part of a building represented by such a small bTOID would not be self-contained so it would make little sense to model it separately.

An examination of the database showed that a given premises could be linked to single or multiple bTOIDs, and vice versa. In some cases the relationships were more complex: a bTOID could be linked to a premises which is linked to several bTOIDs, some of which are linked to further premises and so on. Each set of bTOIDs and premises linked in this way was allocated to a "Group". So a Group consists of those bTOIDs and premises that are interconnected. Each member of a Group is linked to at 
least one other member, and no links extend outside the Group. 7,742 Groups were found in the database as shown in Table 1.

\section{Table 1 near here}

Groups were found to be useful for two reasons. First, they provide a simple conceptual basis for studying the relationships in the LLPG. The analysis is primarily about links among the data items. Since by definition no item in a Group has links with any items outside it, the Group defines the items that need to be studied together. Second, Groups reduce the combinatorial problem of working with large numbers of objects. In particular, it is much easier to determine spatial relationships like thermal contact among a Group of six objects than among 16,000.

A useful way of visualizing Groups is to plot simple examples on an $(\mathrm{x}, \mathrm{y})$ grid, where $\mathrm{x}$ and $\mathrm{y}$ represent the premises and bTOID reference numbers as shown in Fig. 2. Each filled square represents a record in the LLPG database, linking a premises to a bTOID. The square nearest the origin represents a link between premises no. 11 and bTOID no. 100. There are no other entries in the same row or column, that is, with the same bTOID or premises reference number. So neither bTOID nor premises is linked to any other record, and the record is the only member of a Group.

The adjacent Group consists of two records with the same bTOID reference number, 101, and two different premises reference numbers, 12 and 13 , forming a horizontal bar in the diagram. The next Group again 
consists of two records with a common reference number, this time the premises number 14, linked to different bTOID numbers, 102 and 103, resulting in a vertical bar in the diagram.

The final Group consists of links between both bTOIDs and premises, so that the cell with bTOID reference no. 105 and premises no. 15 is linked to other records by both numbers.

Figure 2 near here

Groups were assigned to four different "Classes" depending on the nature of their links. The examples above are representative of each of the four Classes.

4,410 of the Groups (comprising 5,361 records) were in Classes 1 and 2 which means they consist of one bTOID linked to one (Class 1) or more (Class 2) premises.

Since the premises in the single bTOIDs of the Class 1 and 2 Groups do not extend beyond them, these Groups are SCUs according to the definition. They contain single bTOIDs, so the number of SCUs is equal to the number of Groups, namely 3,921 $+489=4,410$. If the assumptions made earlier are correct then they can reasonably be identified with buildings.

The remainder, 3,332 Groups containing 19,109 records, are in Classes 3 and 4. They contain multiple bTOIDs linked to single (Class 3 ) or multiple 
(Class 4) premises. For these cases, further work is required to apply the SCU definition.

\subsection{Spatial relationships between bTOIDs}

A Group as defined above is a set of linked bTOIDs and premises. In a sense, each acts as a "glue" to join entities of the other type, and the analysis so far has treated bTOIDs and premises as equivalent.

However, there are some differences. There are spatial relationships between bTOIDs but not between premises: it is meaningful to ask whether two bTOIDs are in thermal contact, but that is not true of premises. So one interesting feature of Groups is that they can contain sets of bTOIDs which are in physical contact but isolated from other sets within the Group.

This an important issue in the light of the definition of an SCU. A Group defines a set of bTOIDs which are linked by premises and which have no premises in common with other bTOIDs. If, within this Group, sets of bTOIDs are identified that are in thermal contact, in almost all cases they will be SCUs according to the definition (there are some unusual configurations that make it theoretically possible for these contiguous sets to contain more than one SCU, though none was found in the Leicester data).

The further step was therefore made in the data analysis of identifying bTOIDs in thermal contact within groups. Note that it is quite possible for a 
bTOID to be isolated within its Group but attached to a bTOID outside the Group: isolation within a Group does not imply total isolation. The point is to identify the contiguity relations between bTOIDs within each Group in order to apply the definition of an SCU.

The earlier analysis shows that SCUs can be defined immediately for Class 1 and 2 Groups. Defining SCUs in Class 3 and 4 Groups requires more work because the spatial relationships between the bTOIDs comprising the Groups need to be analysed. This was done using specially-written software that determined whether or not the bTOIDs within the Group were in thermal contact, defined as sharing at least 50 cm of party wall. "Contact matrices" were then produced for each Group, indicating which bTOIDs were in thermal contact. Further routines in the software analysed the contact matrices to automatically identify the SCUs in each Group, allowing a total for Classes 3 and 4 to be determined of 5,886 . Accepting for now the equivalence of SCUs and buildings, this allowed, for the first time, the total number of non-domestic buildings in Leicester (other than shopping centres and any categories missing from the Rating List) to be determined. The result was $4,410+5,886=10,296$.

\section{Potential of the SCU concept}

In this section, examples are presented that indicate the potential, and some limitations, of the proposed correspondence between SCUs and buildings. 
A simple example is presented in Fig. 3, which shows an isolated row of three adjoining bTOIDs in the centre of Leicester.

Figure 3 near here

The LLPG shows that the two bTOIDs on the left are occupied by a single premises, while the bTOID to the right contains a further premises.

Application of the SCU definition leads to the boundaries shown by heavy lines in the figure. Comparison with reality shows that the left-hand SCU corresponds to a pub comprising two distinct but similarly-aged sections while the right-hand SCU was a shop and premises which has since gone out of business.

A more complex example is presented in Figure 4 which shows the Ordnance Survey MasterMap representation of part of De Montfort University in Leicester. The buildings marked 1, 2 and 3 are, respectively, the Queens Building, the Kimberlin Library and the Eric Wood Building. A single premises includes all three buildings, but the main bTOID of Building 2 is also linked to a distinct premises within the building: a bookshop. The Group that the linked premises and bTOIDs form is therefore Class 4.

Figure 4 near here

A close examination of the map reveals that Building 3 comprises two bTOIDs while there are eight bTOIDs in Building 2 (two large and six small) and a similar number in Building 1. 
Application of the method described above leads to the automatic identification of three SCUs consistent with the three buildings described above. The complete physical separation of Building 3 from the others demonstrates how the requirement for contiguity of the component bTOIDs leads to the definition of a separate building.

The definition of Buildings 1 and 2 is, however, a little more problematical. It turns out that some of the smaller bTOIDs are absent from the LLPG. In most cases this has no serious consequences, although it does suggest that the SCU definition process adopted should include a comparison stage to check for the existence of such missing elements. But the absence of one particular bTOID does have an impact. This represents a bridge connecting the two buildings, and if it had appeared in the LLPG, Buildings 1 and 2 would have been defined as a single SCU.

On the face of it, this appears to represent a disadvantage in the proposed method. However, the bridge referred to was built precisely to allow Building 1 to be presented as a "Library extension", thus avoiding some administrative difficulties. So the presence of the missing bTOID would in this case have led to agreement with the official status of these buildings. 


\section{Discussion}

\subsection{Status of the work}

The key point about the proposed definition of space is that it comes against a background of very limited alternatives. Individual surveys of non-domestic buildings would allow the bTOIDs that comprise them in digital maps to be identified, but this is an unaffordable luxury for city-scale studies, as in the present case where the number of premises exceeds 10,000

Other possibilities include modelling city blocks comprising all contiguous bTOIDs; studying a small area of a city in detail and assuming the results can be applied elsewhere; and modelling the business premises rather than the building, thus forsaking a physical basis for the modelling approach (Steadman et al 2000).

In this light, our proposed approach could enable an otherwise impracticable modelling task. In this paper we concentrate on demonstrating the reasonableness of the approach given the scale of the task and its potential to cover a very large proportion of a typical urban setting.

7.2 Reasonableness of equating SCUs to buildings

The proposed approach can be broken down into two processes: the definition of SCUs and the equating of SCUs to buildings. 
The definition of SCUs appears to be reasonably robust. No occasions have been discovered when the concept fails, though it is possible to conceive of combinations of premises and bTOIDs that make necessary a slightly more complex approach to the computational definition of SCUs than that described above. In practice, it was found that a simple test within the software could identify such cases.

As things stand, then, a technique that gathers together groups of contiguous bTOIDs in a well defined way for collective analysis seems unremarkable.

The aspect that makes the work interesting is the claim that SCUs are comparable to buildings. If correct, this means that assumptions about the building structure and operation based on the expected uniformity within a building can be applied with a reasonable degree of confidence, raising the possibility of reasonable accuracy from a model even when some detailed data are unavailable.

The principle of the correspondence between SCUs and buildings is that premises that extend from one bTOID to a contiguous one indicate that both bTOIDs are likely to be part of the same building as usually understood.

While this seems reasonable, it is likely that there will be cases where it does not apply. One that was identified early in the work was the shopping centre. On the one hand, shopping centres are sometimes defined as 
single bTOIDs (Neffendorf et al 2009, p. 15). On the other, if separate bTOIDs are defined for each retail outlet, as in the two shopping centres in Leicester, the one-to-one links with the premises suggest that they are distinct buildings. In some ways this is true, but within a larger structure. In addition, the existence of distinct "buildings" on upper and lower floors is also inconsistent with the basic assumption that the bTOIDs are valid at all heights unless they specifically represent overhanging structures, etc. These differences from the standard assumptions for buildings make it clear that shopping centres and comparable structures should be dealt with as special cases outside the SCU framework.

It is likely that the SCU concept also fails occasionally on a smaller scale. For example, it is possible to envisage a business centre comprising several bTOIDs in which one bTOID contains a single premises corresponding to the company renting the space. In such a case the bTOID would be defined as a separate building. Three questions arise: how often this happens, how much it matters, and whether a modification to the definition of an SCU could fix the problem.

On the first point, no examples have been seen as yet, though an exhaustive analysis of the data has not been carried out. On the second, the indications are that any problems would be minor. Party walls with other SCUs would still be treated as such, and the treatment of differences in temperatures on opposite sides of the wall would be dealt with as correctly as the physical modelling allowed. So the matter is mainly one of 
convenience: more calculation would be carried out and more SCUs represented than strictly necessary, but other than that there are no obvious disadvantages. In view of this finding, changes to the SCU definition seem unnecessary.

An interesting outcome of the application of the SCU concept to the data for Leicester was that the number of SCUs is similar to the number of premises. This does not seem unreasonable but there is no obvious reason for it: some buildings (e.g. office blocks) will contain several premises while some premises will extend over several buildings (e.g. hospitals). So the similarity may simply be accidental.

\subsection{Problems and issues}

The most significant issue with the new approach is its dependence on the, presumably manual, work carried out by Council staff to link premises to bTOIDs for the LLPG. Obviously this work was not done specifically to provide the basis for the present work, and it is possible that variations or errors which are minor in the context of the intended outcome could have a more significant effect on the deductions about SCUs.

In this context, it is worth mentioning that further analysis of the Class 4 Groups defined earlier provide possible opportunities for quality checks, though this is not directly relevant to the present work. On a similar note, the consistency between local authorities of the linking methods used will have an impact on the applicability of the SCU concept across the UK. 
Limitations of the method include its restriction to the UK because it requires digital mapping data, business taxation data and the links between them. Further limitations arise as a result of the relatively volatile nature of the taxation data. Changes in the existence or extent of business premises will have an impact on the related SCUs. In particular, if a business ceases to exist, resulting in its disappearance from the VOA database, the corresponding SCU will no longer be defined. This represents a clear difference between the SCU and the building it purports to represent. There is no obvious solution to this problem, although retaining historical VOA entries to ensure the SCU definition continues seems a reasonable approach provided the building is tagged as empty and is modelled as such.

\subsection{Next steps}

The modelling process following successful definition of SCUs has introduced a further set of challenges, mainly relating to the availability and quality of data. In order to minimize such issues a reduced data set approach has been used, although the SCU concept should be valid for any physical modelling method. For the full implementation, internal gains will be derived from the work of Liddiard (2012) while approximate methods will be required for properties of the building fabric, such as the glazing fraction correlations of Gakovic (2000) and the derivations from building age of Smith (2009), assuming age data can be obtained or estimated. 
A trial deployment of a city-scale energy model using approximate data has been carried out using a 3-D visualization tool City View. Figure 5 shows how a single SCU comprising several contiguous bTOIDs containing a single premises is represented in the model.

\section{Figure 5 near here}

The result for an area of about $4 \mathrm{~km}^{2}$ near the centre of Leicester is shown in Fig. 6. Non-domestic buildings, defined as SCUs using the methods described above, are shown as 3D polygons of varying heights, with colour variation representing the estimated annual energy consumption. The lowest consumption is represented by violet and the highest by red. White means no value is available, typically due to a lack of input data for the building energy model.

\section{Figure 6 near here}

The intended outcome of the modelling of Leicester's non-domestic stock is an understanding of the impact on $\mathrm{CO}_{2}$ emissions of a range of interventions such as wall insulation, improved glazing and shading devices, more efficient electrical lighting, connection to district heating and provision of local renewable energy generation (Lomas et al 2010). 


\section{Conclusions}

A new definition of space in the urban built environment, the self-contained unit (SCU), can be derived automatically from the LLPG and equates reasonably well to the concept of building. Its use allows the possibility of city-scale modelling based on the characteristics of individual buildings.

When applied to the city of Leicester, reasonable results were obtained, making possible for the first time an estimate of the number of nondomestic buildings in the city of just over 10,000. This represents a significant advance and further work will be required to demonstrate the power of the approach fully. It must be recognised that the complexity of the urban environment and the data relating to it mean that inevitably there will be caveats, but these are not expected to seriously undermine the usefulness of this new concept in urban scale energy modelling;

Problems identified with the approach include the following:

- It cannot be applied to shopping centres and similar structures which must therefore be dealt with as special cases;

- The SCU depends in a non-audited way on the work carried out to create the LLPG;

- An SCU cannot be defined if a building is unoccupied;

- The concept of SCU is only usable where an LLPG (and its supporting data sources) exists and therefore only applies to the UK. 


\section{Acknowledgments}

The authors acknowledge support by the Engineering and Physical Sciences Research Council (EPSRC) under their Sustainable Urban Environment programme (grant EP/F007604/1) to the 4M consortium: 'Measurement, modelling, mapping and management: an evidence based methodology for understanding and shrinking the urban carbon footprint'. The consortium has five partners in the UK: Loughborough University, De Montfort University, Newcastle University, the University of Sheffield and the University of Leeds. Thanks are due to Ruth Hamilton of Leicester City Council for supplying the LLPG and explaining its structure.

\section{References}

4M (2013). 4M project website

http://mmmm.lboro.ac.uk/. Accessed 14 Jan 2013.

Bruhns, H. R., Steadman, P., Herring, H., Moss, S., \& Rickaby, P. A. (2000). Types, numbers, and floor areas of nondomestic premises in England and Wales, classified by activity. Environment and Planning B: Planning and Design, 27, 641-665.

Bruhns, H., Steadman, P., \& Marjanovic, L. (2006). A Preliminary Model of Non-Domestic Energy Use for England and Wales. COBRA 2006, University College London, 7-8 September 2006 (pp. 7-8). 
Crown Estate (2013). The Crown Estate website

http://www.thecrownestate.co.uk. Accessed 14 Jan 2013.

Gakovic, B. (2000). Areas and types of glazing and other openings in the nondomestic building stock. Environment and Planning B: Planning and Design 27, 667-694.

Hinnells, M., Layberry, R., Curtis, D. and Shea, A. (2008). Transforming UK non-residential buildings: achieving a $60 \%$ cut in $\mathrm{CO} 2$ emissions by 2050. Paper to IEECB, Frankfurt, April 08.

HM Government (2011). The Carbon Plan: Delivering our low carbon future, December 2011. Available at:

http://www.decc.gov.uk/assets/decc/11/tackling-climate-change/carbonplan/3702-the-carbon-plan-delivering-our-low-carbon-future.pdf (Accessed 21 December 2012)

Huang, J., Akbari, H. and Ritschard, R. (1991) 481 Prototypical Commercial Buildings for 20 Urban Market Areas. California, USA: Lawrence Berkeley Laboratory.

Huang, Y. J. and Brodrick, J. (2000) A bottom-up engineering estimate of the aggregate heating and cooling loads of the entire U.S. building stock. California, USA: Lawrence Berkeley Laboratory. 
Jones, P., Williams, J. and Lannon, S. (2000) Planning for a sustainable city: An energy and environmental prediction model. Journal of Environmental Planning and Management, 43, 855-872.

Liddiard, R., Taylor, S., Rylatt, M. (2010). Characterising space use and electricity consumption in non-domestic buildings. Proceedings of IESD PhD Conference: Energy and Sustainable Development. Institute of Energy and Sustainable Development, De Montfort University, Leicester, UK, 21 May 2010.

Liddiard, R.G. (2012). Characterising Space Use and Electricity Consumption in Non-domestic Buildings. PhD thesis, De Montfort University.

Lomas, K.J., Bell, M.C., Firth, S.K., Gaston, K.J., Goodman, P., Leake, J.R., Namdeo, A., Rylatt, M., Allinson, D., Davies, Z.G., Edmondson, J.L., Galatioto, F., Brake, J.A., Guo, L., Fill, G., Irvine, K.N., Taylor, S.C., Tiwary, A. (2010). The carbon footprint of UK Cities: 4M: measurement, modelling, mapping and measurement. ISOCARP Review 06, Int. Soc. of City and Regional Planners, pp. 168-191.

Min, J., Hausfather, Z., Lin, Q.F. (2010). High-Resolution Statistical Model of Residential Energy End Use Characteristics for the United States. Journal of Industrial Ecology 14, 685-862. 
Mortimer, N.D., Ashley, A. and Rix, J.H.R. (2000a) Detailed energy surveys of nondomestic buildings. Environment and Planning B: Planning and Design, 27, 25-32.

Mortimer, N.D., Elsayed, M.A. and Grant, J.F. (2000b). Patterns of energy use in nondomestic buildings. Environment and Planning B: Planning and Design, 27, 709-720.

Neffendorf, H., Bruhns, H.R., Harrison, A. (2009). Non-domestic Energy Efficiency Data Framework (NEED): Scoping Study Findings. Report prepared by Katalysis Ltd for DECC. Available at:

http://www.decc.gov.uk/assets/decc/what\%20we\%20do/supporting\%20co nsumers/saving energy/esdirective/data framework/needreport090129.pdf. Accessed 14 Jan 2013.

OS (2001). OS MasterMap real-world object catalogue v1.0, Ordnance Survey, Nov 2001.

OS (2013). Ordnance Survey website.

http://www.ordnancesurvey.co.uk/oswebsite/products/osmastermap/index.html. Accessed 14 Jan 2013.

Pout, C. H. (2000). N-DEEM: The national nondomestic buildings energy and emissions model. Environment and Planning B: Planning and Design, 27, 721-732. 
Smith, S.T. (2009). Modelling thermal loads for a non-domestic building stock. Associating a priori probability with building form and construction using building control laws and regulations. PhD Thesis, Nottingham University.

Steadman, P., Bruhns, H.R., Rickaby, P.A. (2000). An introduction to the national Non-Domestic Building Stock database. Environment and Planning B: Planning and Design 27, 3-10.

Yamaguchi, Y., Shimoda, Y. and Mizuno, M. (2007) Proposal of a modelling approach considering urban form for evaluation of city level energy management. Energy and Buildings, 39, 580-592.

(7,137 words) 
Tables

Table 1

Analysis of Leicester LLPG

\begin{tabular}{|c|c|c|c|c|c|}
\hline Class & $\begin{array}{c}\text { No. of } \\
\text { Records }\end{array}$ & $\begin{array}{l}\text { No. of } \\
\text { Groups }\end{array}$ & $\begin{array}{c}\text { Records } \\
\text { per Group }\end{array}$ & $\begin{array}{c}\text { No. of } \\
\text { Premises }\end{array}$ & $\begin{array}{l}\text { No. of } \\
\text { bTOIDs }\end{array}$ \\
\hline 1 & 3921 & 3921 & 1.0 & 3921 & 3921 \\
\hline 2 & 1440 & 489 & 2.9 & 1440 & 489 \\
\hline 3 & 9437 & 2745 & 3.4 & 2745 & 9437 \\
\hline 4 & 9672 & 587 & 16.5 & 2756 & 2511 \\
\hline Tota & 24470 & 7742 & 3.2 & 10862 & 16358 \\
\hline
\end{tabular}




\section{Figure captions}

Figure 1. Definition of SCUs.

(a) Some possible arrangements of premises (curves) distributed among bTOIDs (rectangles).

(b) Boundaries of SCUs (dark lines) obtained by applying definition

Figure 2. Types of bTOID/premises links

Figure 3. Application of the SCU concept to a row of bTOIDs in the centre of Leicester.

(C) Crown Copyright/database right 2013. An Ordnance Survey / EDINA supplied service.

Figure 4. Non-domestic buildings in De Montfort University, Leicester (C) Crown Copyright/database right 2013. An Ordnance Survey / EDINA supplied service.

Figure 5. 3-D representation of an SCU consisting of several contiguous bTOIDs linked to a single premises

(C) Crown Copyright/database right 2013. An Ordnance Survey / EDINA supplied service.

Figure 6. A $4 \mathrm{~km}^{2}$ area near the centre of Leicester with non-domestic buildings defined as SCUs and coloured according to energy consumption (C) Crown Copyright/database right 2013. An Ordnance Survey / EDINA supplied service. 
Figures for Taylor et al BRI (2013)

(a)
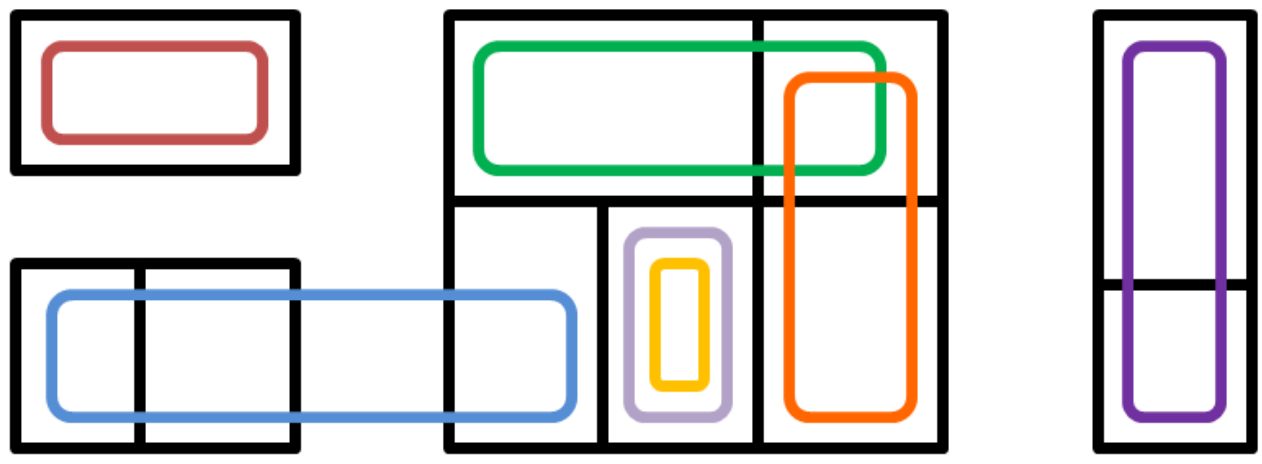

(b)
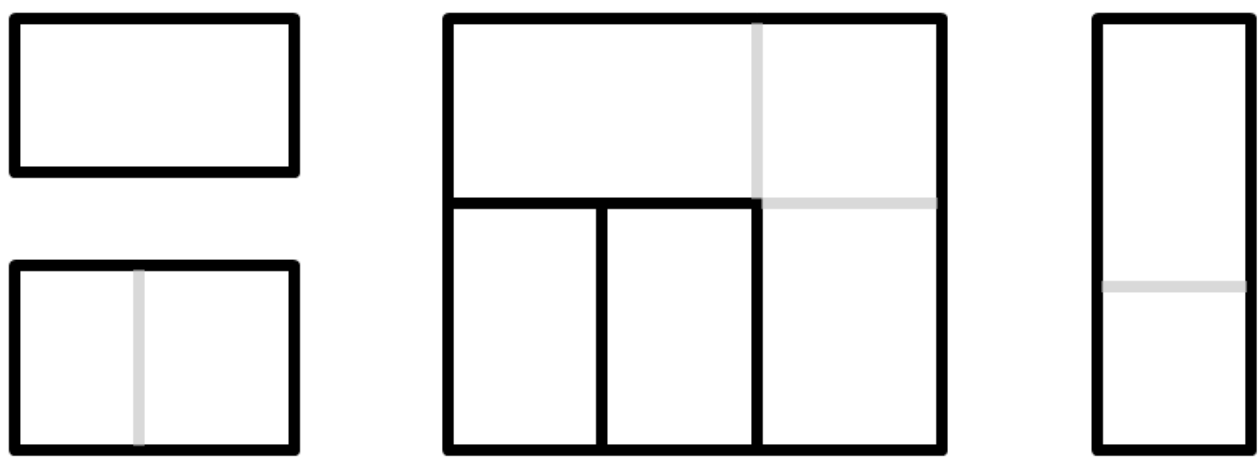

Figure 1 


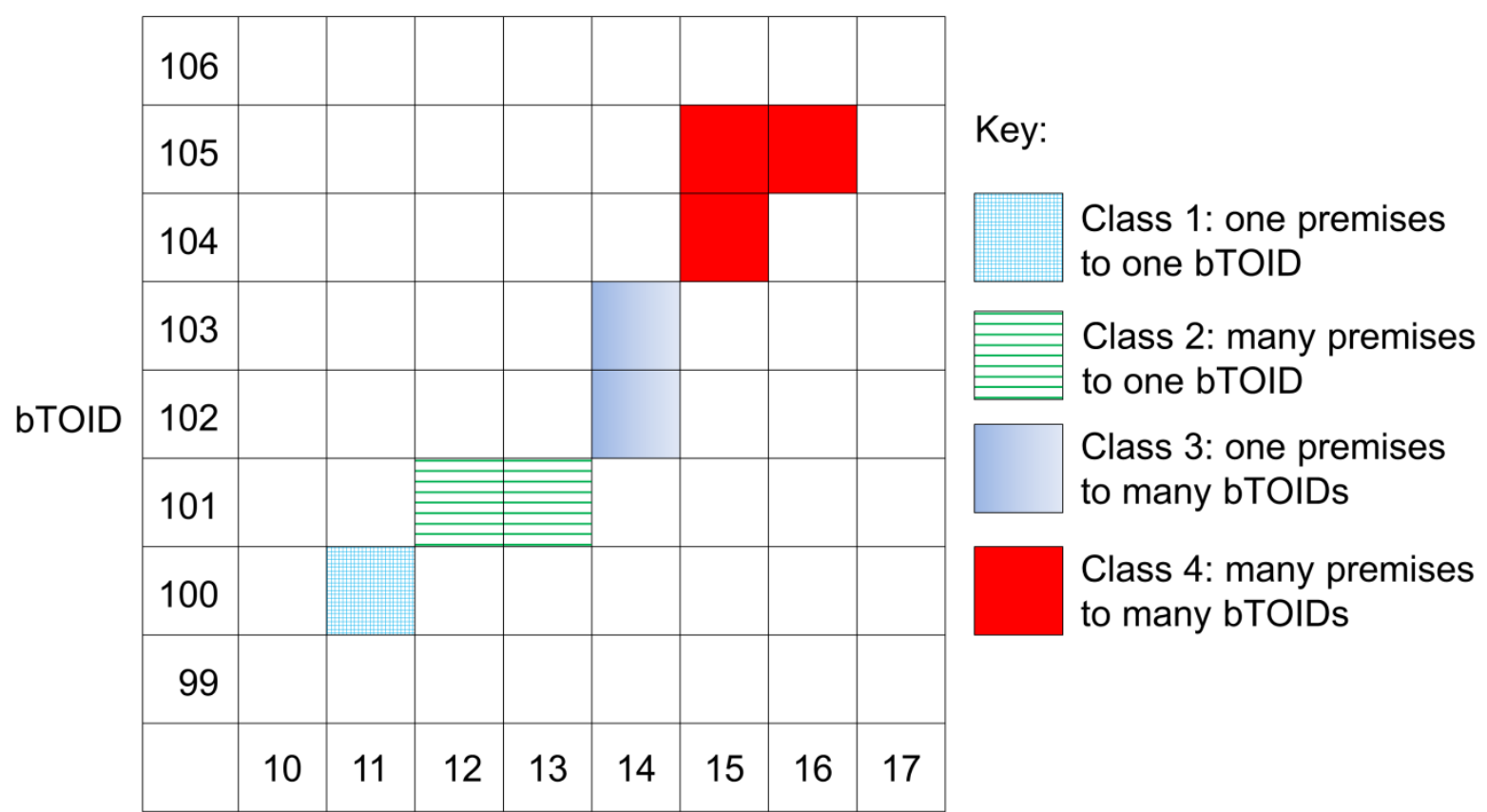

Premises

Figure 2 


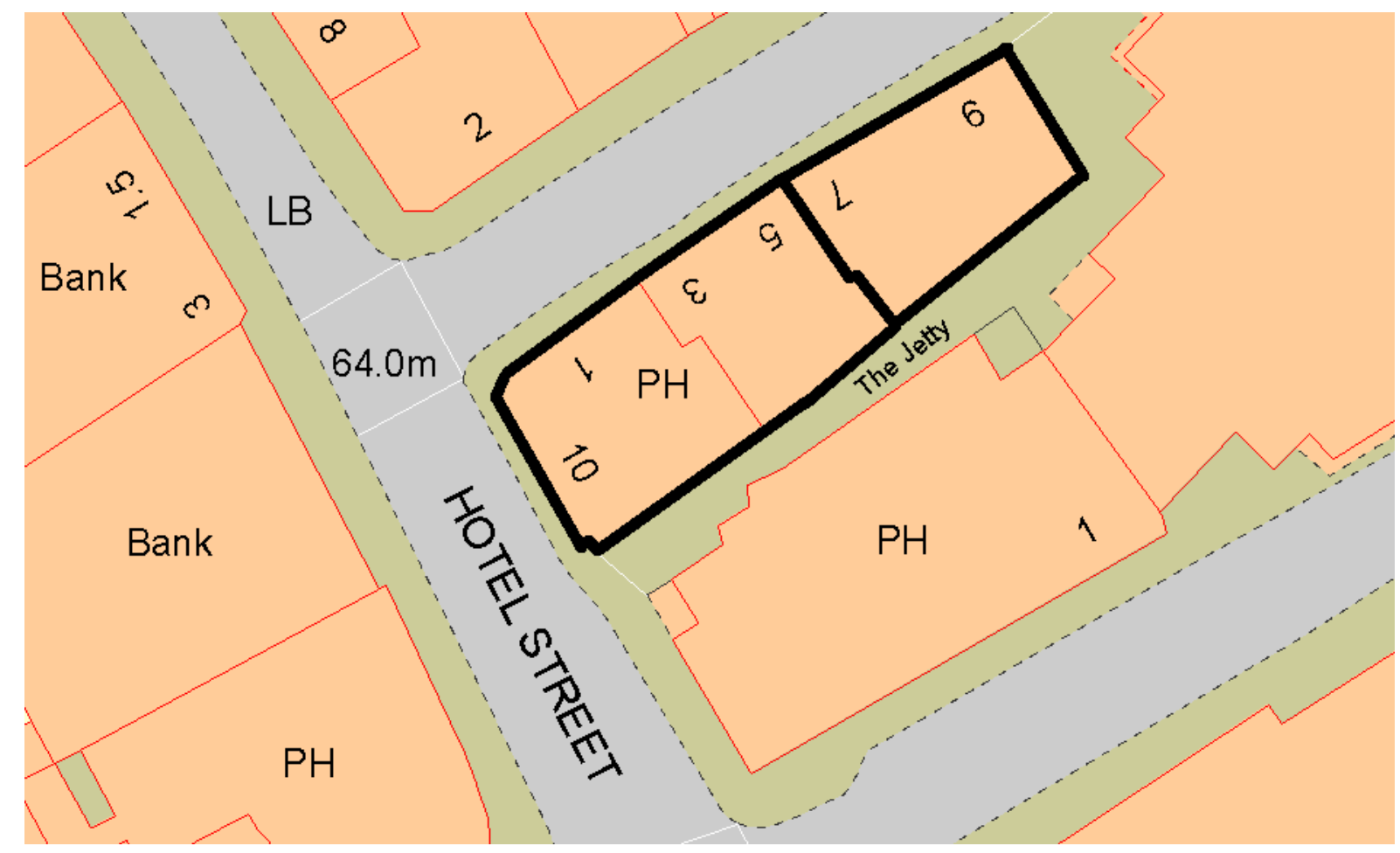

Figure 3

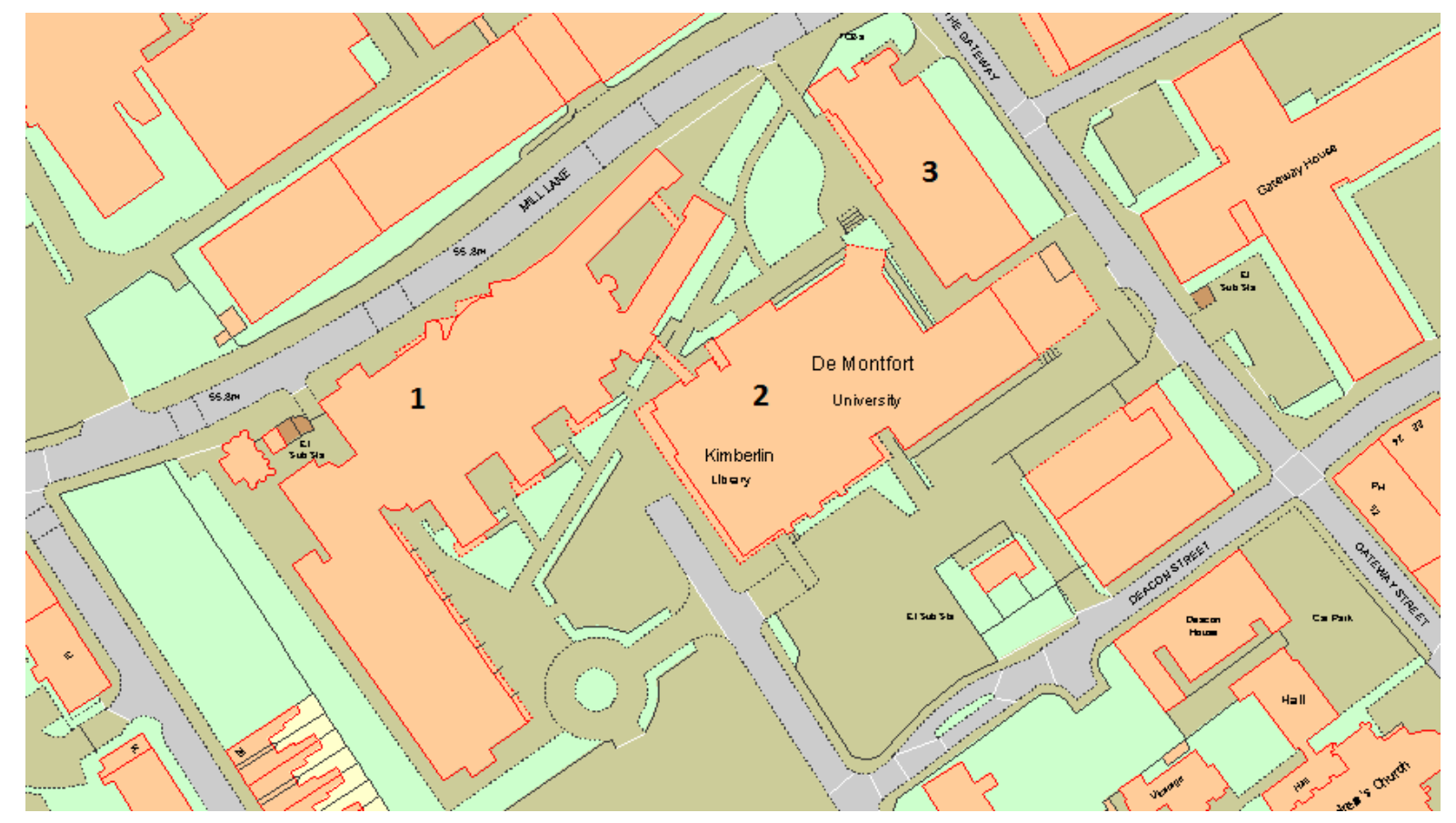

Figure 4 


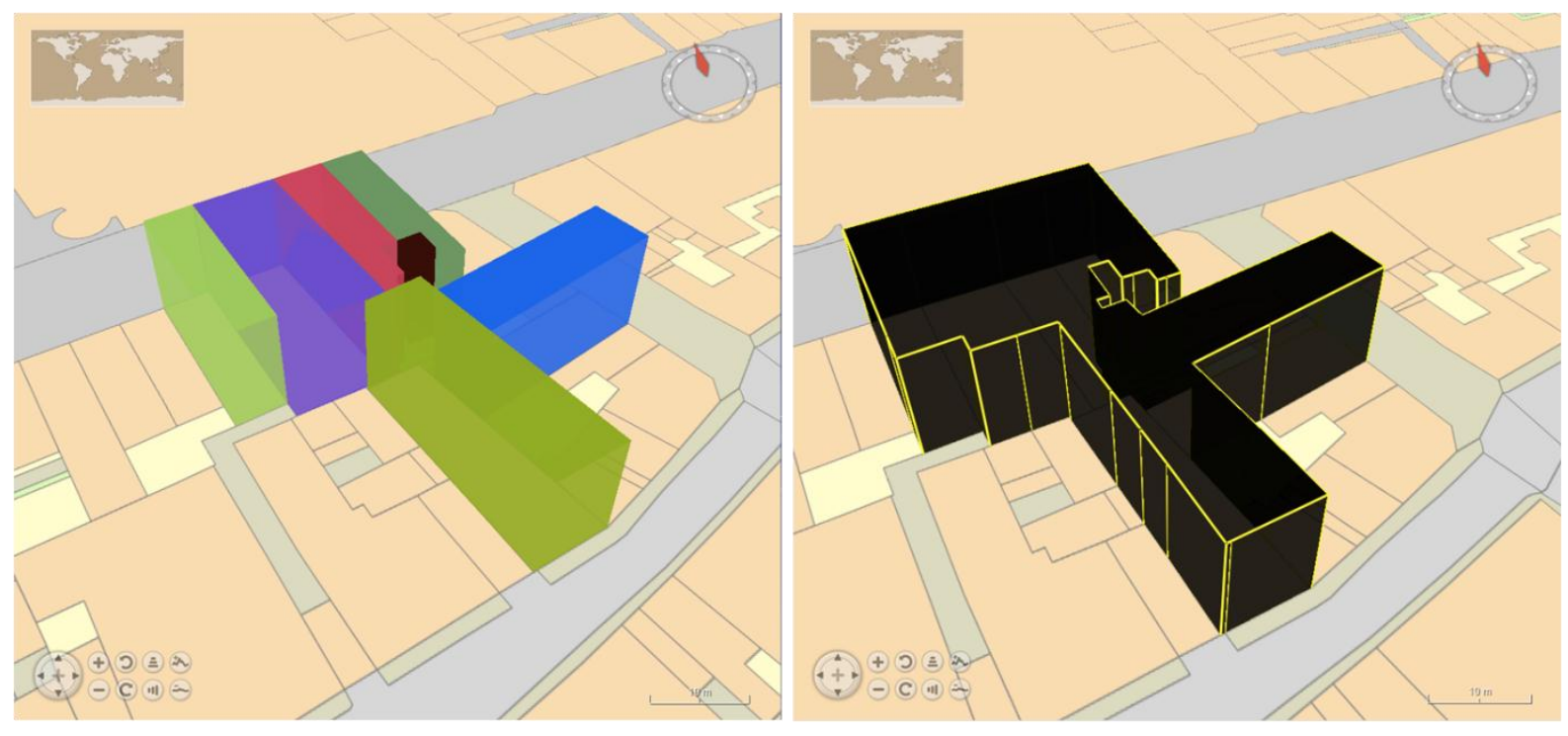

Figure 5
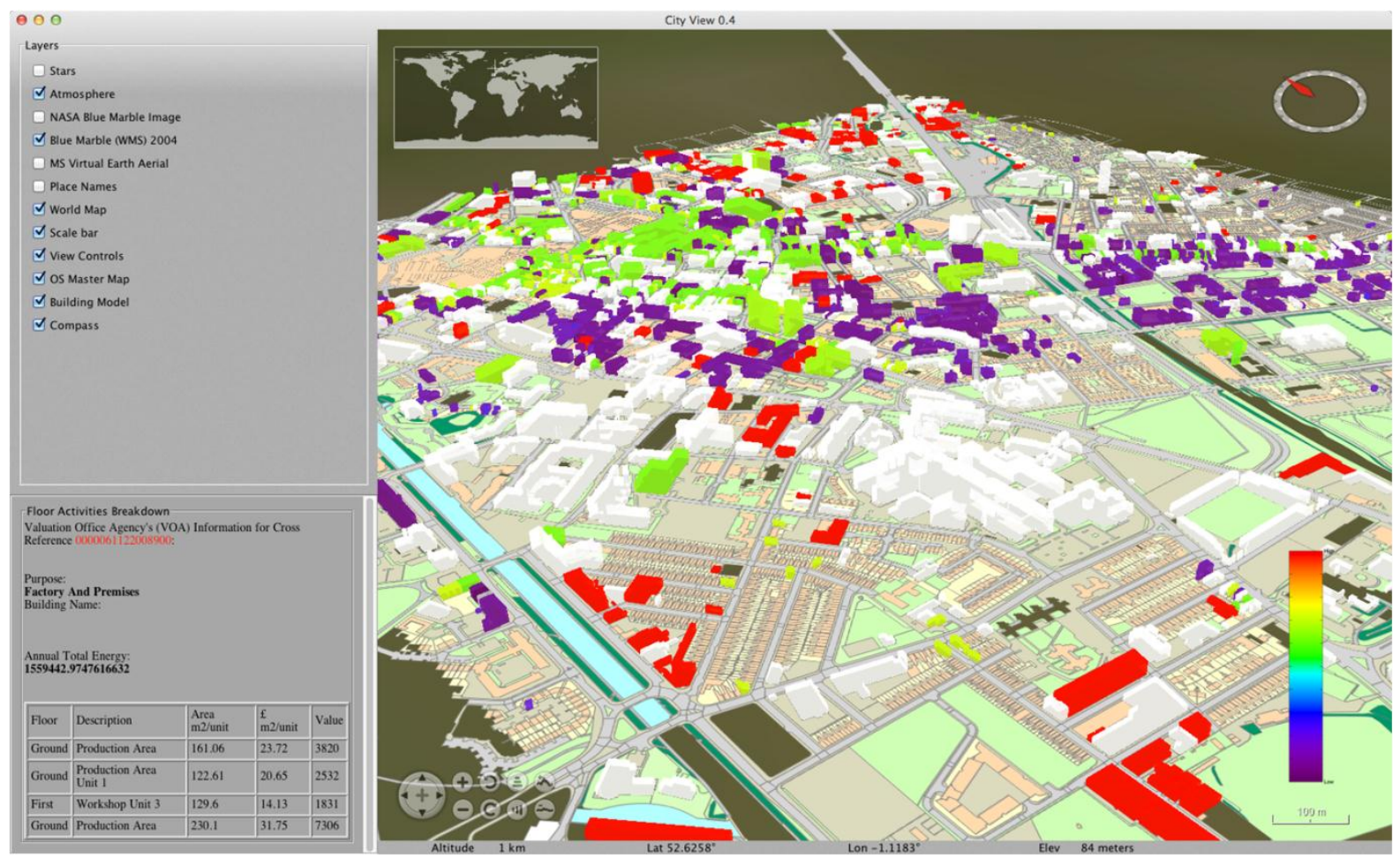

Figure 6 